\title{
ON OPTIMAL SCREENING AND QUARANTINING POLICY IN A NETWORK OF PRISONS
}

\author{
WAI-KI CHING*, YANG CONG ${ }^{\dagger}$, ZHENG-JIAN BAI ${ }^{\ddagger}$, AND TUEN-WAI NG§
}

\begin{abstract}
In this paper, we propose mathematical models for the spread of HIV in a network of prisons. We study the effect of both screening prisoners and quarantining infectives. Efficient algorithms based on Newton's method are then developed for computing the equilibrium values of the infectives in each prison. We also give an optimization formulation for obtaining the optimal screening and quarantine policy. The models and algorithms developed can be extended to model the spread of a disease in a general network of connected zones.
\end{abstract}

Key words: HIV, prison system, epidemic model, equilibrium point, Newton's method, screening policy, quarantine policy.

1. Introduction. Modelling the spread of HIV is an important and interesting topic for a lot of researchers. Many mathematical models have been proposed by different researchers, see for instance Daley and Gani [4], Greenhalgh and Lewis [8], Huang and Villasans [9], Wang [15] and Ma et al. [11]. A serious problem of prison life is the spread of HIV by both sexual contacts and needle sharing activities among the prisoners. In a special report of the New York Times, it was mentioned that the number of HIV infectives in Argentine federal prisons is of the order $30 \%$ of all inmates [6]. Due to the new results in complex networks, now we understand better our social networks [12]. Moreover, people are now connected in efficient transportation networks, dangerous diseases such as SARS can be spread very fast $[3,13]$. Researchers have paid more attention to the spread of diseases in a network [5]. Motivated by the report and the models in [6], Ching et al. [2] proposed a fast numerical algorithm for solving the equilibrium values of the spread of HIV in a network of prisons. However, their model does not consider the impact of screening and quarantining polices. In this paper, we develop models and numerical algorithms for studying such policies as

*Advanced Modeling and Applied Computing Laboratory, Department of Mathematics, The University of Hong Kong, Pokfulam Road, Hong Kong. E-mail: wching@hkusua.hku.hk. Research supported in part by RGC Grants 7017/07P, HKU CRCG Grants, Hung Hing Ying Physical Sciences Research Fund and HKU Strategic Research Theme Fund on Computational Physics and Numerical Methods.

${ }^{\dagger}$ Advanced Modeling and Applied Computing Laboratory, Department of Mathematics, The University of Hong Kong, Pokfulam Road, Hong Kong. E-mail: congyang@hkusua.hku.hk

${ }^{\ddagger}$ Department of Information and Computational Mathematics, Xiamen University, Xiamen 361005, People's Republic of China E-mail: zjbai@xmu.edu.cn. This author's research was partially supported by the National Natural Science Foundation of China Grant 10601043 and Program for New Century Excellent Talents in Xiamen University.

$\S$ Advanced Modeling and Applied Computing Laboratory, Department of Mathematics, The University of Hong Kong, Pokfulam Road, Hong Kong. E-mail: ntw@maths.hku.hk. 
they are useful in reducing the transmission of the disease like HIV in a social network. We remark that the models and algorithms developed can be easily extended to model a spread of a disease in a general network of connected zones.

The paper is structured as follows. In Section 2, we present both discrete time and continuous time models for the spread of HIV in one prison system with screening prisoners and quarantining infectives. We give a sufficient condition for the existence of the system equilibrium. Numerical examples are given to demonstrate the effect of screening prisoners and quarantining infectives. We give a mathematical formulation for finding optimal screening and quarantining policy. In section 3, we extend the model and the theory to a network of prisons. Both discrete time and continuous time models are discussed. Newton's method is then applied to solving the equilibrium values. A sufficient condition is derived for the existence of the inverse of the Jacobian matrix in Newton's method. We also give a discussion on both the stability of the equilibrium and the convergence rate of the Newton's method. Numerical experiments show that Newton's method converges very fast. We then present a mathematical formulation for finding optimal screening and quarantining policy. Finally concluding remarks are given in Section 4.

2. The One-Prison Quarantine Model. We consider a prison consisting of $N$ inmates and it is subject at time $t(t=0,1,2, \ldots)$ to a simultaneous inflow and outflow of $n<N$ prisoners. Then screening and quarantining are taken, after which there are $y(t)$ prisoners who are infected but not identified, $m(t)$ prisoners who are detected HIV positive but not quarantined, $q(t)$ prisoners who are quarantined and $x(t)$ prisoners are susceptible. It is clear that

$$
x(t)=N-y(t)-m(t)-q(t) .
$$

Here we assume that homogeneous mixing occurs in the prison during the interval $(t, t+1)$ and we further assume that the new infectives produced is proportional to $x(t)(y(t)+m(t))$ with a constant $\beta$. This means that the number of new infectives produced is $\beta x(t)(y(t)+m(t))$. Thus at time $t+1$, there are

$$
y(t)+m(t)+\beta x(t)(y(t)+m(t))
$$

infectives not quarantined and there are

$$
y(t)+\beta x(t)(y(t)+m(t))
$$

infectives not detected. For those detected HIV positive, they will be recorded within the prison and will not be screened next time. We also assume that an inflow of $n$ new prisoners from the outside world such that the proportion of infectives is $\mu$. This means that there are $n \mu$ infectives added to the prison. Now a proportion of 
$\tau(0<\tau<1)$ of the prisoners including those incoming prisoners but excluding those quarantined and detected HIV positive will be screened. Then a proportion of $\kappa$ of those detected HIV positive will be quarantined. Now at time $t+1$, after homogeneous mixing, there are $y(t)+\beta x(t)(y(t)+m(t))$ new infectives but not detected prisoners, $m(t)$ detected but not quarantined prisoners. After exchanging with the outside world, there are

$$
\left(1-\frac{n}{N}\right)(y(t)+\beta x(t)(y(t)+m(t)))+n \mu
$$

infected but not detected prisoners, and $\left(1-\frac{n}{N}\right) m(t)$ detected but not quarantined prisoners. We then screen a proportion of $\tau$ of the prisoners. Therefore we get

$$
\begin{aligned}
y(t+1) & =(1-\tau)\left(\left(1-\frac{n}{N}\right)(y(t)+\beta x(t)(y(t)+m(t)))+n \mu\right) \\
& =(1-\tau)\left(1-\frac{n}{N}\right)(y(t)+\beta x(t)(y(t)+m(t)))+(1-\tau) n \mu
\end{aligned}
$$

infected but not detected, and

$$
\left(1-\frac{n}{N}\right) m(t)+\tau\left(\left(1-\frac{n}{N}\right)(y(t)+\beta x(t)(y(t)+m(t)))+n \mu\right)
$$

detected prisoners. A proportion of $\kappa$ of the detected prisoners is then quarantined. We have

$$
\begin{aligned}
m(t+1)= & (1-\kappa)\left(1-\frac{n}{N}\right) m(t) \\
& +(1-\kappa) \tau\left(1-\frac{n}{N}\right)(y(t)+\beta x(t)(y(t)+m(t)))+(1-\kappa) \tau n \mu
\end{aligned}
$$

detected but not quarantined prisoners and

$$
\begin{aligned}
q(t+1)= & \left(1-\frac{n}{N}\right) q(t)+\kappa\left(1-\frac{n}{N}\right) m(t) \\
& +\kappa \tau\left(1-\frac{n}{N}\right)(y(t)+\beta x(t)(y(t)+m(t)))+\tau \kappa \mu n
\end{aligned}
$$

quarantined prisoners. Thus we have the following difference equations:

$$
(4)\left\{\begin{aligned}
y(t+1)= & (1-\tau)\left(1-\frac{n}{N}\right)(y(t)+\beta x(t)(y(t)+m(t)))+(1-\tau) n \mu \\
m(t+1)= & (1-\kappa)\left(1-\frac{n}{N}\right) m(t) \\
& +(1-\kappa) \tau\left(1-\frac{n}{N}\right)(y(t)+\beta x(t)(y(t)+m(t)))+(1-\kappa) \tau n \mu \\
q(t+1)= & \left(1-\frac{n}{N}\right) q(t)+\kappa\left(1-\frac{n}{N}\right) m(t) \\
& +\kappa \tau\left(1-\frac{n}{N}\right)(y(t)+\beta x(t)(y(t)+m(t)))+\tau \kappa \mu n \\
x(t+1)= & N-y(t+1)-m(t+1)-q(t+1) .
\end{aligned}\right.
$$

\subsection{Numerical Demonstration of the Effect of Screening and Quaran-}

tining. We give an example to illustrate the effect of screening and quarantining infected prisoners. We assume

$$
N=500, \beta=0.0005, n=50, y(0)=50, \mu=0.01, \tau=0.1 \text { and } \kappa=0.1 .
$$




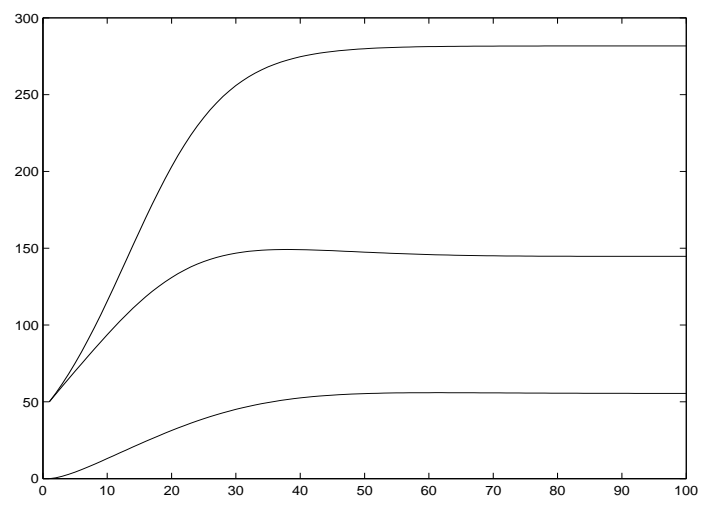

FIG. 1. A Comparison.

In Figure 1, the upper curve represents the number of infectives when there is no screening and quarantining of prisoners (282 infectives in equilibrium state). Suppose a screening policy of $10 \%$ is implemented and among those detected infectives $10 \%$ of them are quarantined. The second curve represents the total number of infectives but not quarantined (145 in equilibrium) and the lowest curve represents the total number of quarantined prisoners (55 in equilibrium). Thus the policy of screening $10 \%$ of the prisoners and quarantining $10 \%$ of the infectives can reduce 82 infectives provided that around $10 \%$ of the space is available for quarantining infected prisoners.

Then in equilibrium, we have

$$
\lim _{t \rightarrow \infty} y(t)=y, \quad \lim _{t \rightarrow \infty} m(t)=m, \quad \lim _{t \rightarrow \infty} q(t)=q, \quad \lim _{t \rightarrow \infty} x(t)=x .
$$

Here we have

(5) $\left\{\begin{aligned} y & =(1-\tau)\left(1-\frac{n}{N}\right)(y+\beta x(y+m))+(1-\tau) n \mu \\ m & =(1-\kappa)\left(1-\frac{n}{N}\right) m+(1-\kappa) \tau\left(1-\frac{n}{N}\right)(y+\beta x(y+m))+(1-\kappa) \tau n \mu \\ \frac{n}{N} q & =\kappa\left(1-\frac{n}{N}\right) m+\kappa \tau\left(1-\frac{n}{N}\right)(y+\beta x(y+m))+\tau \kappa \mu n \\ x & =N-y-m-q .\end{aligned}\right.$

Let $z=y+\beta x(y+m)$, we have

$$
\left\{\begin{array}{l}
y-(1-\tau)\left(1-\frac{n}{N}\right) z=(1-\tau) n \mu \\
\left(\kappa+\frac{n}{N}-\frac{\kappa n}{N}\right) m-(1-\kappa) \tau\left(1-\frac{n}{N}\right) z=(1-\kappa) \tau n \mu \\
\frac{n}{N} q-\kappa\left(1-\frac{n}{N}\right) m-\kappa \tau\left(1-\frac{n}{N}\right) z=\tau \kappa \mu n \\
q+x+y+m=N
\end{array}\right.
$$


Simplifying the equations in (6), we have

$$
\text { (7) }\left\{\begin{array}{l}
y=(1-\tau) n \mu+(1-\tau)\left(1-\frac{n}{N}\right) z \equiv a_{1}+b_{1} z \\
m=\frac{(1-\kappa) \tau n \mu}{\kappa+\frac{n}{N}-\frac{\kappa n}{N}}+\left(\frac{(1-\kappa) \tau\left(1-\frac{n}{N}\right)}{\kappa+\frac{n}{N}-\frac{\kappa n}{N}}\right) z \equiv a_{2}+b_{2} z \\
q=\tau \kappa \mu N+\frac{\tau \mu \kappa(1-\kappa)\left(1-\frac{n}{N}\right) N}{\kappa+\frac{n}{N}-\frac{\kappa n}{N}}+\frac{N}{n}\left(\frac{\kappa \tau(1-\kappa)\left(1-\frac{n}{N}\right)^{2}}{\kappa+\frac{n}{N}-\frac{\kappa n}{N}}+\kappa \tau\left(1-\frac{n}{N}\right)\right) z \\
\equiv a_{3}+b_{3} z \\
x=\frac{z-y}{\beta(y+m)}
\end{array}\right.
$$

Using the fact that $x+y+m+q=N$, we have

$$
\left(a_{1}+a_{2}+a_{3}\right)+\left(b_{1}+b_{2}+b_{3}\right) z+\frac{z-a_{1}-b_{1} z}{\beta\left(\left(a_{1}+a_{2}\right)+\left(b_{1}+b_{2}\right) z\right)}=N .
$$

We get the following equation:

$$
\begin{aligned}
\beta\left(a_{1}+a_{2}\right) N+\beta\left(b_{1}+b_{2}\right) N z= & \beta\left(a_{1}+a_{2}+a_{3}\right)\left(\left(a_{1}+a_{2}\right)+\left(b_{1}+b_{2}\right) z\right) \\
& +\beta\left(a_{1}+a_{2}\right)\left(b_{1}+b_{2}+b_{3}\right) z \\
& +\beta\left(b_{1}+b_{2}+b_{3}\right)\left(b_{1}+b_{2}\right) z^{2}+\left(1-b_{1}\right) z-a_{1} .
\end{aligned}
$$

Thus we obtain a quadratic equation:

$$
A z^{2}+B z+C=0
$$

where

$$
\left\{\begin{array}{l}
A=\beta\left(b_{1}+b_{2}\right)\left(b_{1}+b_{2}+b_{3}\right) \\
B=1-b_{1}+\beta\left(\left(a_{1}+a_{2}\right)\left(b_{1}+b_{2}+b_{3}\right)+\left(b_{1}+b_{2}\right)\left(\sum_{i=1}^{3} a_{i}-N\right)\right) \\
C=\beta\left(a_{1}+a_{2}\right)\left(\sum_{i=1}^{3} a_{i}-N\right)-a_{1} .
\end{array}\right.
$$

Solving $z$, one can solve the equilibrium solution. We have the following proposition and the proof can be found in Appendix.

Proposition 1. A sufficient condition for the quadratic equation (9) to have a unique positive root (therefore the existence of the equilibrium point of (4)) is

$$
\mu<\frac{N}{2 N+n}
$$

From the results of the discrete time model, it is straightforward to develop the 
model to the continuous case as follows:

$$
\left\{\begin{aligned}
\frac{d y(t)}{d t}= & \left((1-\tau)\left(1-\frac{n}{N}\right)-1\right) y(t) \\
& +(1-\tau)\left(1-\frac{n}{N}\right) \beta x(t)(y(t)+m(t))+(1-\tau) \mu n \\
\frac{d m(t)}{d t}= & \left((1-\kappa)\left(1-\frac{n}{N}\right)-1\right) m(t) \\
& +\tau(1-\kappa)\left(1-\frac{n}{N}\right)(y(t)+\beta x(t)(y(t)+m(t)))+(1-\kappa) \tau \mu n \\
\frac{d q(t)}{d t}= & -\frac{n}{N} q(t)+\kappa\left(1-\frac{n}{N}\right) m(t) \\
& +\kappa \tau\left(1-\frac{n}{N}\right)(y(t)+\beta x(t)(y(t)+m(t)))+\tau \kappa \mu n \\
\frac{d x(t)}{d t}= & -\frac{d y(t)}{d t}-\frac{d m(t)}{d t}-\frac{d q(t)}{d t} .
\end{aligned}\right.
$$

In equilibrium, we have

$$
\frac{d y(t)}{d t}=\frac{d m(t)}{d t}=\frac{d q(t)}{d t}=\frac{d x(t)}{d t}=0 .
$$

It is straightforward to show that

Proposition 2. The equilibrium solutions of (10) satisfy the equations in (5).

Since the analysis of the continuous model in our context is similar to the discrete model, we will focus on the discrete model only. For the stability of the equilibrium, we have the following result and the proof can be found in Appendix.

Proposition 3. Under the condition of Proposition 1, the equilibrium point of (4) determined by (9) is asymptotically stable.

We end this subsection by presenting the numerical results of equilibrium solutions when $N=500, \beta=0.0005, n=50$, and $\mu=0.01$ for different values of $\tau$ and $\kappa$ in Figures 2 and 3.

2.2. Optimal Screening and Quarantining Policy. In this subsection, we consider the problem of finding the optimal screening and quarantining policy. In order to reduce the number of infectives in the system, screening prisoners and quarantining infectives are effective strategies. However, there are costs associated with these strategies. Here we assume that the screening cost $C_{s}(\tau, N)$ depends on $\tau$ and $N$ only and the quarantining $\operatorname{cost} C_{q}(\kappa, N)$ depends on $\kappa$ and $N$ only. Then the total cost is given by

$$
C(\tau, \kappa, N)=C_{s}(\tau, N)+C_{q}(\kappa, N) .
$$

For the screening cost and the quarantining cost, one may assume that

$$
C_{s}(\tau, N)=a \tau N \quad \text { and } \quad C_{q}(\kappa, N)=b \kappa N
$$

for some positive constants $a$ and $b$. At the same time, there are some constraints to be met. One would expect that the number of quarantined prisoners $q$ in equilibrium 

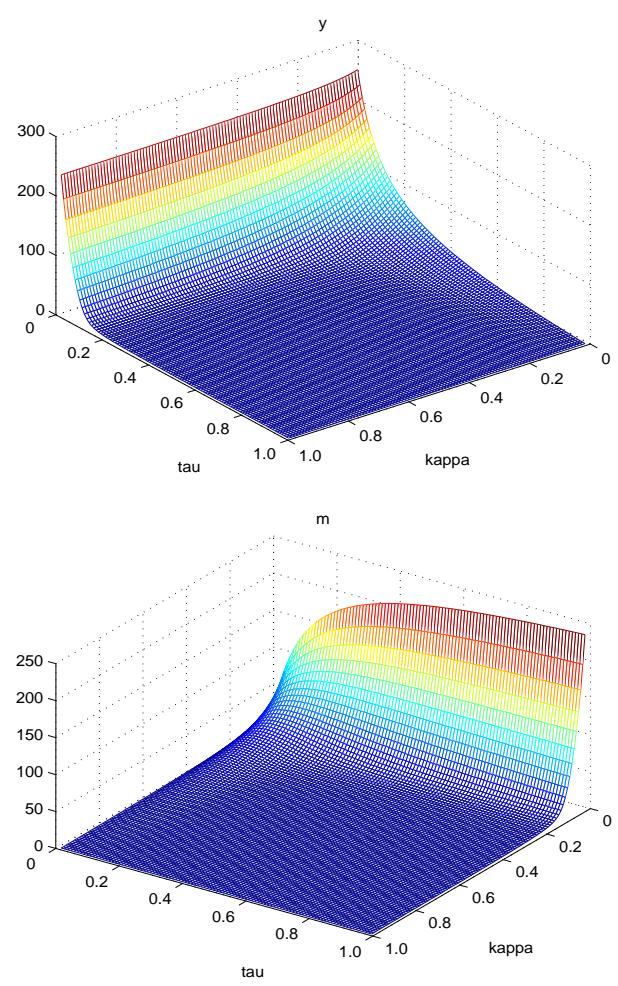

FIG. 2. Total infected prisoners but not (detected (Left)) (quarantined (Right)).

cannot exceed an upper physical limit $Q$ and the total number of infectives $y+m+q$ is less than or equal to a certain tolerant level $I$. Therefore one may consider the following optimization problem:

$$
\begin{cases} & \min C(\tau, \kappa, N)=N(a \tau+b \kappa) \\ \text { s.t. } & \\ & q \leq Q \\ & y+m+q \leq I \\ & 0 \leq \tau, \kappa \leq 1 .\end{cases}
$$

Here $q, m, y$ are the equilibrium solutions when $\tau, \kappa, N$ are given.

To find the optimal values of $\tau$ and $\kappa$, one may perform a grid search, say with grid size 0.01 for both parameters. This means that we are going to try all possible pairs $(\tau, \kappa)$ of the form:

$$
\{(0.01 i, 0.01 j): i, j=0,1, \ldots, 100\} .
$$

For each pair of $(\tau, \kappa)$, we solve for the equilibrium values and therefore the constraints can be checked and the cost function can be evaluated. Hence we can obtain the optimal pair $(\tau, \kappa)$ up to two decimal places. 

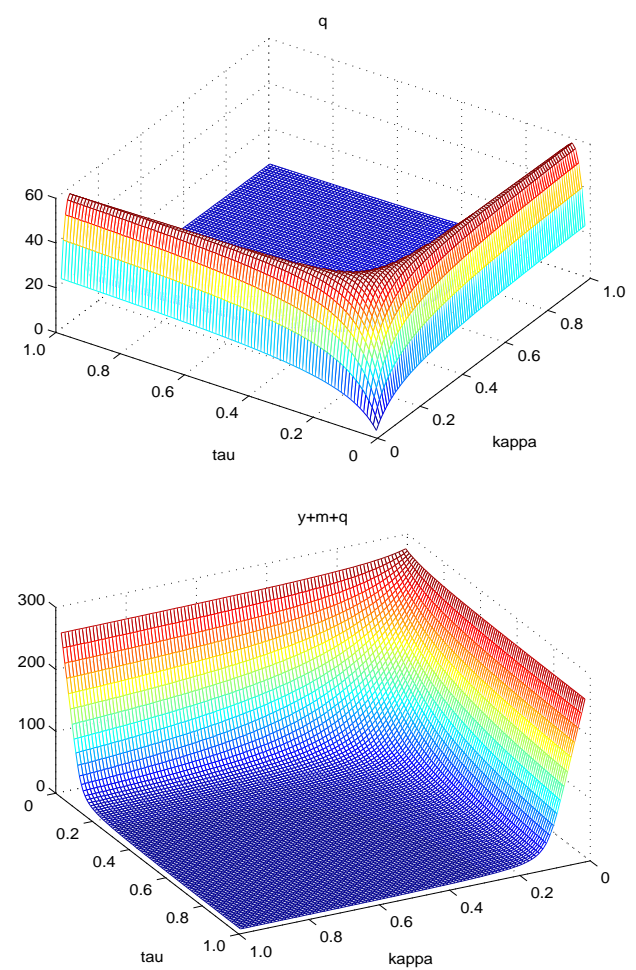

FIG. 3. Total quarantined prisoners (Left) and infectives (Right).

In the following, we suppose that $N=500, \beta=0.0005, n=50, \mu=0.01$ and $a=100, b=500, Q=30, I=100$. The first optimal pair is $(0.38,0.15)$, the equilibrium values are given as follows:

$$
(y, m, q, y+m+q)=(7.5,16.7,29.6,53.8)
$$

and the second optimal pair $(0.43,0.14)$ and the equilibrium values are given as follows:

$$
(y, m, q, y+m+q)=(6.4,18.4,30.0,54.8) .
$$

The optimal cost in both cases are the same as 56500 .

3. A Network of Prisons. In this section, we consider the case of a network of $s$ prisons. We assume there are interactions among the prisons and also with the outside world. Let us give the notations of the model as follows:

(i) $N_{i}$, the number of prisoners in Prison $i$.

(ii) $y_{i}(t)$, the number of prisoners who are infected but not tested at time $t$ in Prison $i$. 
(iii) $m_{i}(t)$, the number of prisoners who are detected HIV positive but not quarantined at time $t$ in Prison $i$.

(iv) $q_{i}(t)$, the number of prisoners who are quarantined at time $t$ in Prison $i$.

(v) $\beta_{i}$, the infection rate in Prison $i$.

(vi) $\mu$, the mean proportion of infectives in the outside world.

(vii) $n_{i}$, the number of prisoners in Prison $i$, exchanging with the outside world.

(viii) $h$, the number of prisoners exchange between the two prisons.

It is clear that for $i=1,2, \ldots, s$

$$
x_{i}(t)=N_{i}-y_{i}(t)-m_{i}(t)-q_{i}(t)
$$

is the number of susceptible prisoners in Prison $i$. The discrete model is then given as follows:

$$
\left\{\begin{aligned}
y_{i}(t+1)= & (1-\tau)\left(1-\frac{n_{i}+(s-1) h}{N_{i}}\right)\left(y_{i}(t)+\beta_{i} x_{i}(t)\left(y_{i}(t)+m_{i}(t)\right)\right) \\
& +(1-\tau) \sum_{j=1, j \neq i}^{s} \frac{h}{N_{j}}\left(y_{j}(t)+\beta_{j} x_{j}(t)\left(y_{j}(t)+m_{j}(t)\right)\right)+(1-\tau) n_{i} \mu \\
m_{i}(t+1)= & (1-\kappa)\left(1-\frac{n_{i}+(s-1) h}{N_{i}}\right) m_{i}(t)+(1-\kappa) \sum_{j=1, j \neq i}^{s} \frac{h}{N_{j}} m_{j}(t) \\
& +(1-\kappa) \tau\left(1-\frac{n_{i}+(s-1) h}{N_{i}}\right)\left(y_{i}(t)+\beta_{i} x_{i}(t)\left(y_{i}(t)+m_{i}(t)\right)\right) \\
& +(1-\kappa) \tau \sum_{j=1, j \neq i}^{s} \frac{h}{N_{j}}\left(y_{j}(t)+\beta_{j} x_{j}(t)\left(y_{j}(t)+m_{j}(t)\right)\right)+(1-\kappa) \tau n_{i} \mu \\
q_{i}(t+1)= & \left(1-\frac{n_{i}+(s-1) h}{N_{i}}\right) q_{i}(t)+\sum_{j=1, j \neq i}^{s} \frac{h}{N_{j}} q_{j}(t)+\kappa\left(1-\frac{n_{i}+(s-1) h}{N_{i}}\right) m_{i}(t) \\
& \left.+\kappa \sum_{j=1, j \neq i}^{s} \frac{h}{N_{j}} m_{j}(t)\right)+\kappa \tau\left(1-\frac{n_{i}+(s-1) h}{N_{i}}\right)\left(y_{i}(t)+\beta_{i} x_{i}(t)\left(y_{i}(t)+m_{i}(t)\right)\right) \\
& +\kappa \tau \sum_{j=1, j \neq i}^{s} \frac{h}{N_{j}}\left(y_{j}(t)+\beta_{j} x_{j}(t)\left(y_{j}(t)+m_{j}(t)\right)\right)+\kappa \tau n_{i} \mu
\end{aligned}\right.
$$

For the equilibrium point, similarly we use

$$
z_{i}=y_{i}+\beta_{i} x_{i}\left(y_{i}+m_{i}\right), \quad i=1,2, \ldots, s .
$$


Then we can get

(13)

$$
\left\{\begin{aligned}
y_{i}= & (1-\tau)\left(1-\frac{n_{i}+(s-1) h}{N_{i}}\right) z_{i}+(1-\tau) \sum_{j=1, j \neq i}^{s} \frac{h}{N_{j}} z_{j}+(1-\tau) n_{i} \mu=0 \\
m_{i}= & (1-\kappa)\left(1-\frac{n_{i}+(s-1) h}{N_{i}}\right) m_{i}+(1-\kappa) \sum_{j=1, j \neq i}^{s} \frac{h}{N_{j}} m_{j} \\
& +(1-\kappa) \tau\left(1-\frac{n_{i}+(s-1) h}{N_{i}}\right) z_{i} \\
& +(1-\kappa) \tau \sum_{j=1, j \neq i}^{s} \frac{h}{N_{j}} z_{j}+(1-\kappa) \tau n_{i} \mu=0 \\
q_{i}= & \left(1-\frac{n_{i}+(s-1) h}{N_{i}}\right) q_{i}+\sum_{j=1, j \neq i}^{s} \frac{h}{N_{j}} q_{j}+\kappa\left(1-\frac{n_{i}+(s-1) h}{N_{i}}\right) m_{i} \\
& +\kappa \sum_{j=1, j \neq i}^{s} \frac{h}{N_{j}} m_{j}+\kappa \tau\left(1-\frac{n_{i}+(s-1) h}{N_{i}}\right) z_{i} \\
& +\kappa \tau \sum_{j=1, j \neq i}^{s} \frac{h}{N_{j}} z_{j}+\kappa \tau n_{i} \mu=0 \\
z_{i}= & y_{i}+\beta_{i} x_{i} y_{i}+\beta_{i} x_{i} m_{i}=0 .
\end{aligned}\right.
$$

To find the condition such that the equilibrium solution determined by (13) is asymptotically stable, we first discuss the case of the network of $s$ prisons without prisoners exchange between the two prisons, i.e., $h=0$. By setting $h=0$ in (13), we obtain

$$
\left\{\begin{aligned}
y_{i} & =(1-\tau)\left(1-\frac{n_{i}}{N_{i}}\right) z_{i}+(1-\tau) n_{i} \mu \\
m_{i} & =(1-\kappa)\left(1-\frac{n_{i}}{N_{i}}\right) m_{i}+(1-\kappa) \tau\left(1-\frac{n_{i}}{N_{i}}\right) z_{i}+(1-\kappa) \tau n_{i} \mu \\
q_{i} & =\left(1-\frac{n_{i}}{N_{i}}\right) q_{i}+\kappa\left(1-\frac{n_{i}}{N_{i}}\right) m_{i}+\kappa \tau\left(1-\frac{n_{i}}{N_{i}}\right) z_{i}+\kappa \tau n_{i} \mu \\
z_{i} & =y_{i}+\beta_{i} x_{i} y_{i}+\beta_{i} x_{i} m_{i}
\end{aligned}\right.
$$

By a simplification, (14) becomes

$$
\left\{\begin{aligned}
y_{i} & =(1-\tau) n_{i} \mu+(1-\tau)\left(1-\frac{n_{i}}{N_{i}}\right) z_{i} \equiv a_{i 1}^{o}+b_{i 1}^{o} z_{i} \\
m_{i} & =\frac{(1-\kappa) \tau n_{i} \mu}{\kappa+\frac{n_{i}}{N_{i}}-\frac{\kappa n_{i}}{N_{i}}}+\frac{(1-\kappa) \tau\left(1-\frac{n_{i}}{N_{i}}\right)}{\kappa+\frac{n_{i}}{N_{i}}-\frac{\kappa n_{i}}{N_{i}}} z_{i} \equiv a_{i 2}^{o}+b_{i 2}^{o} z_{i} \\
q_{i} & =\kappa \tau \mu N_{i}+\frac{N_{i}}{n_{i}} \kappa\left(1-\frac{n_{i}}{N_{i}}\right) a_{i 2}^{o}+\frac{N_{i}}{n_{i}} \kappa\left(1-\frac{n_{i}}{N_{i}}\right)\left(b_{i 2}^{o}+\tau\right) z_{i} \equiv a_{i 3}^{o}+b_{i 3}^{o} z_{i} \\
z_{i} & =y_{i}+\beta_{i} x_{i} y_{i}+\beta_{i} x_{i} m_{i} .
\end{aligned}\right.
$$

On the existence of an equilibrium solution to (14), we have the following sufficient condition. Based on (15), the proof is similar to that of Proposition 1, we omit it here.

Proposition 4. There exists an equilibrium solution to (14) if the following condition holds

$$
\mu<\frac{N_{i}}{2 N_{i}+n_{i}} \quad \text { for } \quad i=1,2, \ldots, s
$$


For the stability of the equilibrium point determined by (14), we have the following result whose proof can be found in Appendix.

Proposition 5. Under the conditions of Proposition 4, the equilibrium point of the discrete model for a network of s prisons without prisoners exchange between the two prisons is asymptotically stable.

Now, we consider the general case for the network of $s$ prisons, where there exists prisoners exchange between the two prisons, i.e., $h>0$. For $i=1, \ldots, s$, let

$$
\left\{\begin{array}{l}
c_{i}=\kappa+\frac{n_{i}+(s-1) h}{N_{i}}-\frac{\kappa\left(n_{i}+(s-1) h\right)}{N_{i}}, \\
d_{i}=\frac{n_{i}+(s-1) h}{N_{i}} .
\end{array}\right.
$$

By a simple calculation, we can reduce (13) to the following form

$$
\left\{\begin{aligned}
y_{i}= & (1-\tau) n_{i} \mu+(1-\tau)\left(1-\frac{n_{i}+(s-1) h}{N_{i}}\right) z_{i}+(1-\tau) \sum_{j=1, j \neq i}^{s} \frac{h}{N_{j}} z_{j} \\
\equiv & a_{i 1}+b_{i 1} z_{i}+(1-\tau) \sum_{j=1, j \neq i}^{s} \frac{h}{N_{j}} z_{j} \\
m_{i}= & \frac{(1-\kappa) \tau n_{i} \mu}{c_{i}}+\frac{(1-\kappa) \tau\left(1-\frac{n_{i}+(s-1) h}{N_{i}}\right)}{c_{i}} z_{i} \\
& +\frac{(1-\kappa)}{c_{i}} \sum_{j=1, j \neq i}^{s} \frac{h}{N_{j}} m_{j}+\frac{(1-\kappa) \tau}{c_{i}} \sum_{j=1, j \neq i}^{s} \frac{h}{N_{j}} z_{j} \\
\equiv & a_{i 2}+b_{i 2} z_{i}+e_{i} \sum_{j=1, j \neq i}^{s} \frac{h}{N_{j}} m_{j}+f_{i} \sum_{j=1, j \neq i}^{s} \frac{h}{N_{j}} z_{j} \\
q_{i} & \frac{\kappa \tau n_{i} \mu}{d_{i}}+\frac{\kappa\left(1-d_{i}\right)}{d_{i}} a_{i 2}+\frac{\kappa\left(1-d_{i}\right)}{d_{i}}\left(b_{i 2}+\tau\right) z_{i} \\
& +\left(\frac{(1-\kappa) \kappa\left(1-d_{i}\right)}{c_{i} d_{i}}+\frac{\kappa}{d_{i}}\right) \sum_{j=1, j \neq i}^{s} \frac{h}{N_{j}} m_{j} \\
& +\frac{1}{d_{i}} \sum_{j=1, j \neq i}^{s} \frac{h}{N_{j}} q_{j}+\left(\frac{(1-\kappa) \kappa \tau\left(1-d_{i}\right)}{c_{i} d_{i}}+\frac{\kappa \tau}{d_{i}}\right) \sum_{j=1, j \neq i}^{s} \frac{h}{N_{j}} z_{j} \\
\equiv & a_{i 3}+b_{i 3} z_{i}+g_{i} \sum_{j=1, j \neq i}^{s} \frac{h}{N_{j}} m_{j}+h_{i} \sum_{j=1, j \neq i}^{s} \frac{h}{N_{j}} q_{j}+w_{i} \sum_{j=1, j \neq i}^{s} \frac{h}{N_{j}} z_{j} \\
z_{i} & y_{i}+\beta_{i} x_{i} y_{i}+\beta_{i} x_{i} m_{i} .
\end{aligned}\right.
$$

Based on (16), we can establish the stability of the equilibrium point determined by (13). The proof can be found in Appendix.

Proposition 6. Suppose that $\mu<\frac{N_{i}}{2 N_{i}+n_{i}}$ for $i=1, \ldots, s$. The equilibrium point of the discrete model for the network of $s$ prisons is asymptotically stable if $h \ll N_{i}$ for $i=1, \ldots, s$.

REMARK 1. Under the conditions of Proposition 6, the Jacobian matrix is nonsingular. Thus one can use the Newton's method for our problem and the quadratic converge is guaranteed if the initial guess is close to the equilibrium solution suffciently.

In the following numerical experiments, we assume that

$$
N_{1}=N_{2}=500 ; n_{i}=10 i ; h=10 ; y_{1}=y_{2}=100 ; \mu=0.01 .
$$




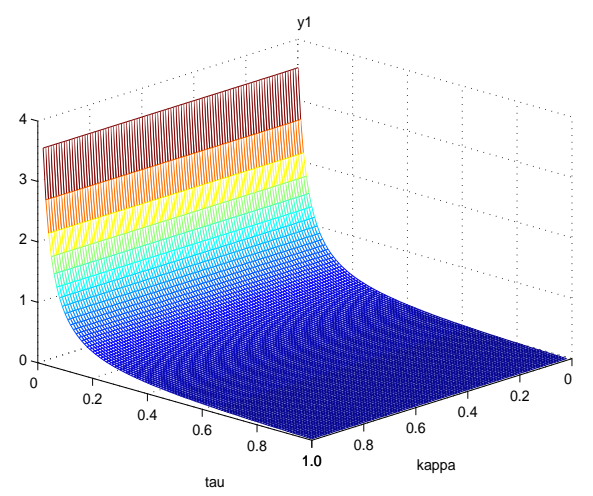

$\mathrm{m} 1$

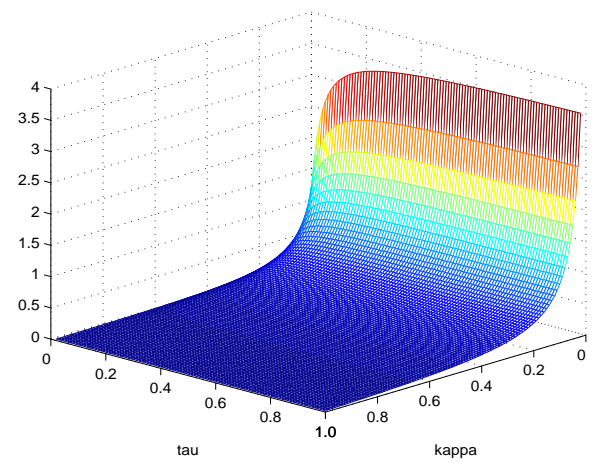

FIG. 4. Total infected prisoners but not (detected (Left)) (quarantined (Right)) in Prison 1.

TABLE 3.1

$\tau=0.03, \kappa=0.07$

\begin{tabular}{lccc}
\hline$\beta_{2}$ & 0.0000003 & 0.000003 & 0.00003 \\
\hline$\left(y_{1}, y_{2}\right)$ & $(2.2,2.7)$ & $(2.2,2.7)$ & $(2.4,3.4)$ \\
$\left(m_{1}, m_{2}\right)$ & $(0.7,0.7)$ & $(0.7,0.7)$ & $(0.8,0.9)$ \\
$\left(q_{1}, q_{2}\right)$ & $(2.1,1.6)$ & $(2.2,1.6)$ & $(2.5,1.9)$ \\
\hline
\end{tabular}

For non-symmetric prisoner system we present the numerical results. We first consider the case when $\beta_{1}=0.0000003$.

We then present the numerical result when we have a bigger $\beta_{1}=0.0003$. From Table 3.1 to Table 3.6, with the increase of $\beta_{2}$, for both prisons, the infected not detected, detected not quarantined and the quarantined all increase. With the increase of the infective rate in Prison 2, the HIV prisoners generated by homogeneous mixing are increased for Prison 2. As a result, $y_{2}, m_{2}, q_{2}$ are increased. Due to the exchange of prisoners between the two prisons, the HIV infectives brought in from Prison 2 increase. 
TABle 3.2

$$
\tau=0.05, \kappa=0.05
$$

\begin{tabular}{lccc}
\hline$\beta_{2}$ & 0.0000003 & 0.000003 & 0.00003 \\
\hline$\left(y_{1}, y_{2}\right)$ & $(1.5,2.1)$ & $(1.5,2.1)$ & $(1.6,2.6)$ \\
$\left(m_{1}, m_{2}\right)$ & $(1.1,1.2)$ & $(1.1,1.2)$ & $(1.2,1.4)$ \\
$\left(q_{1}, q_{2}\right)$ & $(2.4,1.8)$ & $(2.4,1.8)$ & $(2.7,2.1)$ \\
\hline
\end{tabular}

Table 3.3

$$
\tau=0.07, \kappa=0.03
$$

\begin{tabular}{lccc}
\hline$\beta_{2}$ & 0.0000003 & 0.000003 & 0.00003 \\
\hline$\left(y_{1}, y_{2}\right)$ & $(1.2,1.7)$ & $(1.2,1.7)$ & $(1.2,2.1)$ \\
$\left(m_{1}, m_{2}\right)$ & $(1.7,1.7)$ & $(1.7,1.8)$ & $(1.9,2.1)$ \\
$\left(q_{1}, q_{2}\right)$ & $(2.1,1.6)$ & $(2.2,1.6)$ & $(2.5,1.9)$ \\
\hline
\end{tabular}

TABle 3.4

$$
\tau=0.03, \kappa=0.07
$$

\begin{tabular}{lccc}
\hline$\beta_{2}$ & 0.0000003 & 0.000003 & 0.00003 \\
\hline$\left(y_{1}, y_{2}\right)$ & $(3.0,2.9)$ & $(3.0,2.9)$ & $(3.3,3.7)$ \\
$\left(m_{1}, m_{2}\right)$ & $(0.9,0.8)$ & $(1.0,0.8)$ & $(1.1,1.0)$ \\
$\left(q_{1}, q_{2}\right)$ & $(2.7,1.9)$ & $(2.8,1.9)$ & $(3.2,2.3)$ \\
\hline
\end{tabular}

Table 3.5

$$
\tau=0.05, \kappa=0.05
$$

\begin{tabular}{llll}
\hline$\beta_{2}$ & 0.0000003 & 0.000003 & 0.00003 \\
\hline$\left(y_{1}, y_{2}\right)$ & $(2.1,2.2)$ & $(2.1,2.2)$ & $(2.3,2.7)$ \\
$\left(m_{1}, m_{2}\right)$ & $(1.5,1.3)$ & $(1.5,1.3)$ & $(1.6,1.6)$ \\
$\left(q_{1}, q_{2}\right)$ & $(3.0,2.1)$ & $(3.0,2.1)$ & $(3.4,2.5)$ \\
\hline
\end{tabular}

TAble 3.6

$$
\tau=0.07, \kappa=0.03
$$

\begin{tabular}{lccc}
\hline$\beta_{2}$ & 0.0000003 & 0.000003 & 0.00003 \\
\hline$\left(y_{1}, y_{2}\right)$ & $(1.7,1.7)$ & $(1.7,1.8)$ & $(1.8,2.2)$ \\
$\left(m_{1}, m_{2}\right)$ & $(2.3,1.9)$ & $(2.3,2.0)$ & $(2.6,2.4)$ \\
$\left(q_{1}, q_{2}\right)$ & $(2.7,1.9)$ & $(2.8,1.9)$ & $(3.2,2.3)$ \\
\hline
\end{tabular}



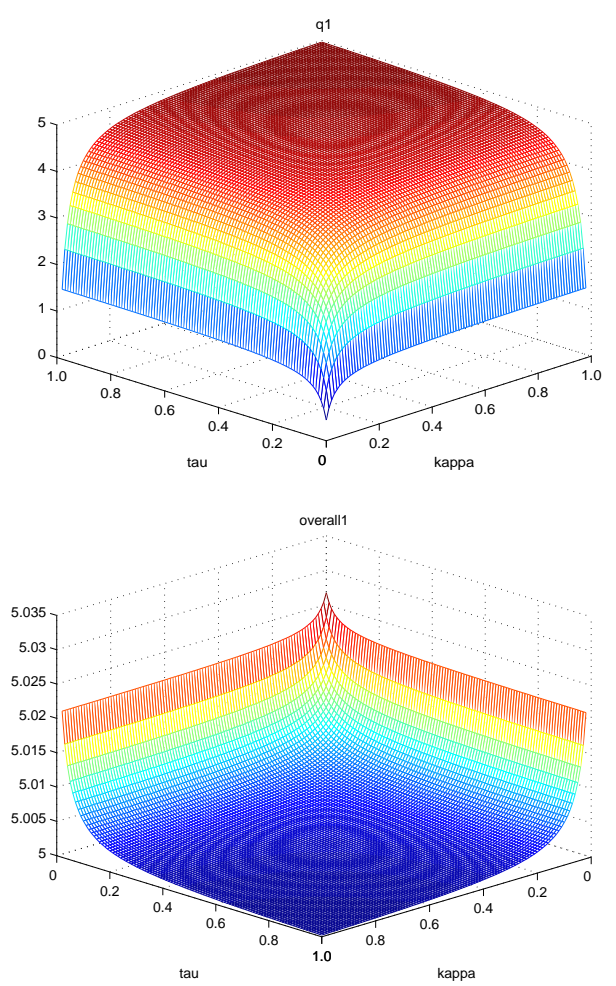

FIG. 5. Total quarantined prisoners (Left) and infectives (Right) in Prison 1.

TABLE 3.7

$$
\tau=0.03, \kappa=0.07
$$

\begin{tabular}{lccccc}
\hline$h$ & 10 & 15 & 20 & 25 & 30 \\
\hline$\left(y_{1}, y_{2}\right)$ & $(2.17,2.68)$ & $(2.22,2.65)$ & $(2.26,2.62)$ & $(2.28,2.60)$ & $(2.31,2.59)$ \\
$\left(m_{1}, m_{2}\right)$ & $(0.71,0.72)$ & $(0.72,0.71)$ & $(0.73,0.71)$ & $(0.73,0.71)$ & $(0.73,0.71)$ \\
$\left(q_{1}, q_{2}\right)$ & $(2.14,1.61)$ & $(2.08,1.66)$ & $(2.03,1.68)$ & $(2.00,1.70)$ & $(1.98,1.72)$ \\
\hline
\end{tabular}

We now discuss the effect of the exchange between the two prisons. In the following numerical experiments, we also assume that

$$
N_{1}=N_{2}=500, n_{i}=10 i, y_{1}=y_{2}=100, \beta_{1}=\beta_{2}=0.0000003 \quad \text { and } \quad \mu=0.01 \text {. }
$$

From Table 3.7 to Table 3.9, the increase of $h$ stands for the increase of prisoners exchanged between the two prison. This would of course lead to the increase of mixing of the prisoners in the two prisons. Thus the differences between the infectives but not detected and the quarantined infectives for both prisons all decrease. 

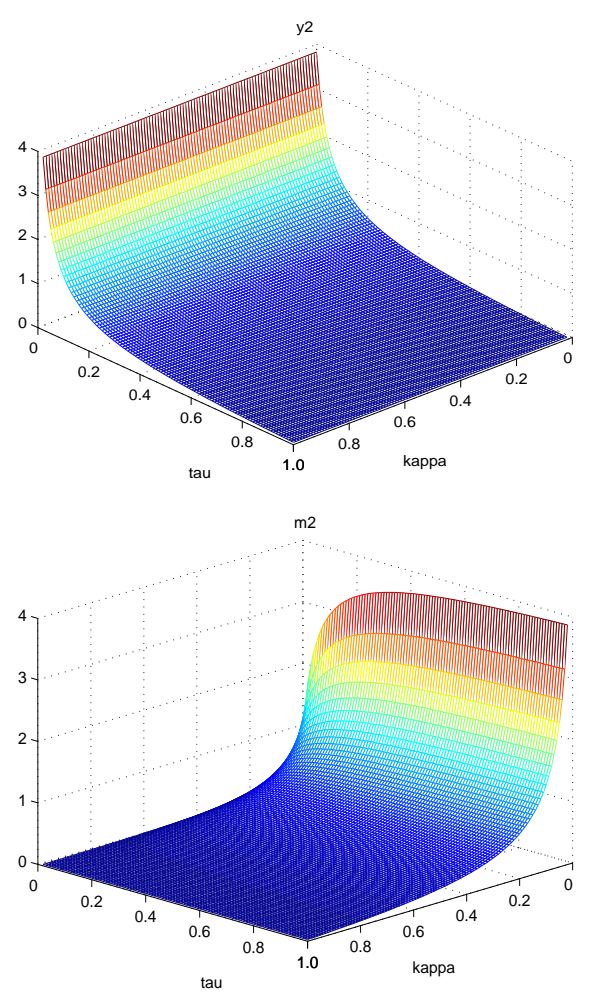

FIG. 6. Total infected prisoners but not (detected (Left)) (quarantined (Right)) in Prison 2.

TABLE 3.8

$$
\tau=0.05, \kappa=0.05
$$

\begin{tabular}{lccccc}
\hline$h$ & 10 & 15 & 20 & 25 & 30 \\
\hline$\left(y_{1}, y_{2}\right)$ & $(1.53,2.05)$ & $(1.57,2.02)$ & $(1.60,1.99)$ & $(1.62,1.97)$ & $(1.64,1.96)$ \\
$\left(m_{1}, m_{2}\right)$ & $(1.12,1.16)$ & $(1.14,1.14)$ & $(1.15,1.14)$ & $(1.16,1.14)$ & $(1.16,1.13)$ \\
$\left(q_{1}, q_{2}\right)$ & $(2.37,1.81)$ & $(2.31,1.85)$ & $(2.26,1.88)$ & $(2.23,1.90)$ & $(2.21,1.92)$ \\
\hline
\end{tabular}

3.1. Optimal Screening and Quarantining Policy. In this subsection, we consider the two-prison case when applying optimal screening and quarantining policy. Here we assume that the screening $\operatorname{cost} C_{s i}\left(\tau_{i}, N_{i}\right)$ depends on $\tau_{i}$ and $N_{i}$ only, the quarantining $\operatorname{cost} C_{q i}\left(\kappa_{i}, N_{i}\right)$ depends on $\kappa_{i}$ and $N_{i}$ only, $i=1,2$. Therefore the total cost is

$$
C=\sum_{i=1}^{2} C\left(\tau_{i}, \kappa_{i}, N_{i}\right)=\sum_{i=1}^{2} C_{s i}\left(\tau_{i}, N_{i}\right)+\sum_{i=1}^{2} C_{q i}\left(\kappa_{i}, N_{i}\right) .
$$

We also assume that for $i=1,2$,

$$
C_{s i}\left(\tau_{i}, N_{i}\right)=a \tau_{i} N_{i} \quad \text { and } \quad C_{q i}\left(\kappa_{i}, N_{i}\right)=b \kappa_{i} N_{i}
$$



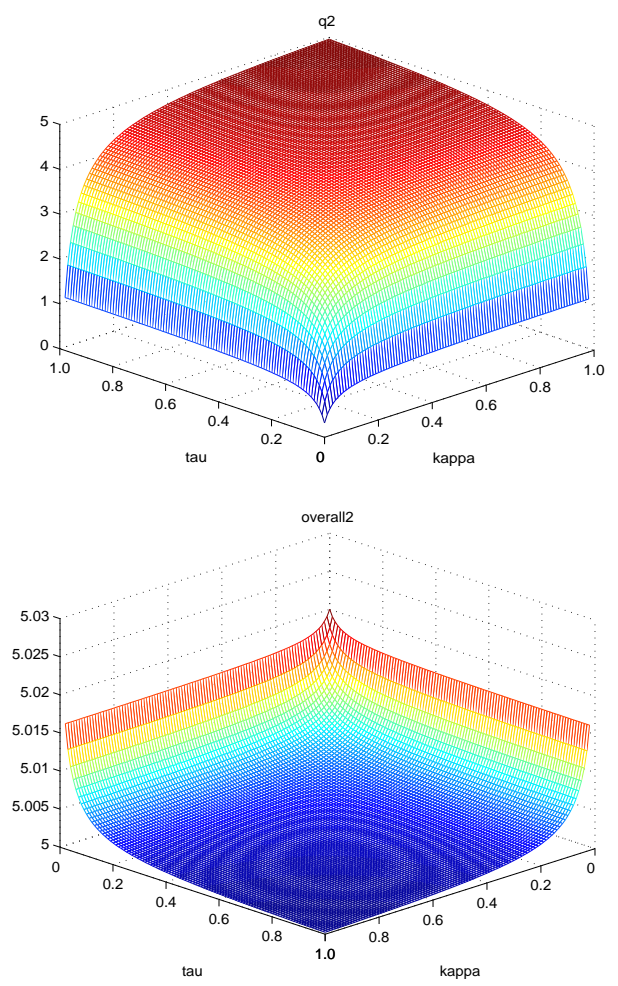

FIG. 7. Total quarantined prisoners (Left) and infectives (Right) in Prison 2.

TABLE 3.9

$$
\tau=0.07, \kappa=0.03
$$

\begin{tabular}{lccccc}
\hline$h$ & 10 & 15 & 20 & 25 & 30 \\
\hline$\left(y_{1}, y_{2}\right)$ & $(1.16,1.65)$ & $(1.19,1.63)$ & $(1.22,1.60)$ & $(1.24,1.59)$ & $(1.25,1.57)$ \\
$\left(m_{1}, m_{2}\right)$ & $(1.72,1.75)$ & $(1.75,1.73)$ & $(1.77,1.73)$ & $(1.78,1.72)$ & $(1.78,1.72)$ \\
$\left(q_{1}, q_{2}\right)$ & $(2.14,1.61)$ & $(2.08,1.66)$ & $(2.03,1.68)$ & $(2.00,1.70)$ & $(1.98,1.72)$ \\
\hline
\end{tabular}

for some positive constants $a$ and $b$. We would like to minimize the above cost but at the same time, there are some constraints to be met. We would expect that the number of quarantined prisoners $q_{i}$ in equilibrium does not exceed an upper physical limit $Q_{i}$ and the total number of infectives $y_{i}+m_{i}+q_{i}$ is less than or equal to a tolerant level $I_{i}, i=1,2$. To simplify the case, we assume that $\tau_{1}=\tau_{2}=\tau$, and 
$\kappa_{1}=\kappa_{2}=\kappa$. Thus the optimization is

$$
\begin{cases} & \min C(\tau, \kappa, N)=(a \tau+b \kappa)\left(N_{1}+N_{2}\right) \\ \text { s.t. } & q_{i} \leq Q_{i}, i=1,2 \\ & \sum_{i=1}^{2} y_{i}+m_{i}+q_{i} \leq I \\ & 0 \leq \tau, \kappa \leq 1 .\end{cases}
$$

Here $q_{i}, m_{i}, y_{i}, i=1,2$ are equilibrium values when $\tau, \kappa, N_{i}, i=1,2$ are given.

To find the optimal values of $\tau$ and $\kappa$, we perform a grid search, say with grid size 0.01 for both parameters. This means we are going to try all possible pairs $(\tau, \kappa)$ of the form $\{(0.01 i, 0.01 j): i, j=0,1, \ldots, 100\}$. For each pair of $(\tau, \kappa)$, we then solve for the equilibrium values and therefore the the constraints can be checked and the cost function can also be evaluated. Hence we can obtain the optimal pair $(\tau, \kappa)$.

In the following numerical example, we suppose that

$$
N_{i}=500, \beta_{i}=0.00003, y(i)=100, i=1,2 n_{1}=10, n_{2}=20, \mu=0.01, h=10
$$

and

$$
a=100, b=500, Q_{1}=Q_{2}=4, I=14 .
$$

We have two optimal pairs $(0.07,0.04)$ and $(0.12,0.03)$. When the optimal pair is $(0.07,0.04)$ is applied, the equilibrium values are given as follows:

$$
\left(y_{1}, m_{1}, q_{1}, y_{1}+m_{1}+q_{1}\right)=(1.7,2.1,3.4,7.3)
$$

and

$$
\left(y_{2}, m_{2}, q_{2}, y_{2}+m_{2}+q_{2}\right)=(2.2,2.0,2.6,6.7) .
$$

When $(0.12,0.03)$ is applied the equilibrium values are given as follows:

$$
\left(y_{1}, m_{1}, q_{1}, y_{1}+m_{1}+q_{1}\right)=(1.0,2.8,3.4,7.3)
$$

and

$$
\left(y_{2}, m_{2}, q_{2}, y_{2}+m_{2}+q_{2}\right)=(1.4,2.8,2.6,6.7) .
$$

The optimal cost in both cases are the same as 27000 .

4. Concluding Remarks. We propose mathematical models for modeling the spread of HIV in a network of prisons with the effect of both screening prisoners and quarantining infectives. Efficient algorithm based on Newton's method was developed for computing the equilibrium values of the infectives in each prison. Optimal screening and quarantine policy can be obtained by solving a simple optimization problem.

Acknowledgment: The authors would like to thank the anonymous referee for many helpful comments, corrections and constructive suggestions. 


\section{Appendix.}

5.1. Proof of Proposition 1. In the following, we analyze the roots of the quadratic equation

$$
A z^{2}+B z+C=0
$$

First, we note that

$$
\kappa+\frac{n}{N}-\frac{\kappa n}{N}=-\left((1-\kappa)\left(1-\frac{n}{N}\right)-1\right)
$$

Thus we know that

$$
\kappa+\frac{n}{N}-\frac{\kappa n}{N}>0
$$

Hence we can get

$$
\left\{\begin{array}{l}
a_{1}=(1-\tau) n \mu>0, \\
0<b_{1}=(1-\tau)\left(1-\frac{n}{N}\right)<1, \\
a_{2}=\frac{(1-\kappa) \tau n \mu}{\kappa+\frac{n}{N}-\frac{\kappa n}{N}>0} \\
b_{2}=\frac{(1-\kappa) \tau\left(1-\frac{n}{N}\right)}{\kappa+\frac{n}{N}-\frac{\kappa n}{N}}>0, \\
a_{3}=\tau \kappa \mu N+\frac{\tau \kappa(1-\kappa)\left(1-\frac{n}{N}\right)}{\kappa+\frac{n}{N}-\frac{\kappa n}{N}}>0, \\
b_{3}=\frac{N}{n}\left(\frac{\kappa \tau(1-\kappa)\left(1-\frac{n}{N}\right)^{2}}{\kappa+\frac{n}{N}-\frac{\kappa n}{N}}+\kappa \tau\left(1-\frac{n}{N}\right)\right)>0 \\
A=\beta\left(b_{1}+b_{2}\right)\left(b_{1}+b_{2}+b_{3}\right)>0 .
\end{array}\right.
$$

Now we discuss the roots of the quadratic equation $A z^{2}+B z+C=0$ by considering $B^{2}-4 A C$.

$$
\begin{aligned}
B^{2}= & \left(1-b_{1}\right)^{2}+\beta^{2}\left(a_{1}+a_{2}\right)^{2}\left(b_{1}+b_{2}+b_{3}\right)^{2}+\beta^{2}\left(b_{1}+b_{2}\right)^{2}\left(\sum_{i=1}^{3} a_{i}-N\right)^{2} \\
& +2\left(1-b_{1}\right) \beta\left(a_{1}+a_{2}\right)\left(b_{1}+b_{2}+b_{3}\right)+2\left(1-b_{1}\right) \beta\left(b_{1}+b_{2}\right)\left(\sum_{i=1}^{3} a_{i}-N\right) \\
(19) \quad & +2 \beta^{2}\left(a_{1}+a_{2}\right)\left(b_{1}+b_{2}+b_{3}\right)\left(b_{1}+b_{2}\right)\left(\sum_{i=1}^{3} a_{i}-N\right) \\
4 A C= & 4 \beta\left(b_{1}+b_{2}\right)\left(b_{1}+b_{2}+b_{3}\right)\left(\beta\left(a_{1}+a_{2}\right)\left(\sum_{i=1}^{3} a_{i}-N\right)-a_{1}\right) \\
= & 4 \beta^{2}\left(b_{1}+b_{2}\right)\left(b_{1}+b_{2}+b_{3}\right)\left(a_{1}+a_{2}\right)\left(\sum_{i=1}^{3} a_{i}-N\right)-4 a_{1} \beta\left(b_{1}+b_{2}\right)\left(b_{1}+b_{2}+b_{3}\right)
\end{aligned}
$$


Then we have

$$
\begin{aligned}
B^{2}-4 A C= & \left(1-b_{1}\right)^{2}+\beta^{2}\left(a_{1}+a_{2}\right)^{2}\left(b_{1}+b_{2}+b_{3}\right)^{2}+\beta^{2}\left(b_{1}+b_{2}\right)^{2}\left(\sum_{i=1}^{3} a_{i}-N\right)^{2} \\
& +2\left(1-b_{1}\right) \beta\left(a_{1}+a_{2}\right)\left(b_{1}+b_{2}+b_{3}\right)+2\left(1-b_{1}\right) \beta\left(b_{1}+b_{2}\right)\left(\sum_{i=1}^{3} a_{i}-N\right) \\
& -2 \beta^{2}\left(a_{1}+a_{2}\right)\left(b_{1}+b_{2}+b_{3}\right)\left(b_{1}+b_{2}\right)\left(\sum_{i=1}^{3} a_{i}-N\right) \\
& +4 a_{1} \beta\left(b_{1}+b_{2}\right)\left(b_{1}+b_{2}+b_{3}\right) \\
= & \left(\left(1-b_{1}\right)^{2}+\beta^{2}\left(a_{1}+a_{2}\right)^{2}\left(b_{1}+b_{2}+b_{3}\right)^{2}+\beta^{2}\left(b_{1}+b_{2}\right)^{2}\left(\sum_{i=1}^{3} a_{i}-N\right)^{2}\right. \\
& -2\left(1-b_{1}\right) \beta\left(a_{1}+a_{2}\right)\left(b_{1}+b_{2}+b_{3}\right)+2\left(1-b_{1}\right) \beta\left(b_{1}+b_{2}\right)\left(\sum_{i=1}^{3} a_{i}-N\right) \\
& \left.-2 \beta^{2}\left(a_{1}+a_{2}\right)\left(b_{1}+b_{2}+b_{3}\right)\left(b_{1}+b_{2}\right)\left(\sum_{i=1}^{3} a_{i}-N\right)\right) \\
& +4\left(1-b_{1}\right) \beta\left(a_{1}+a_{2}\right)\left(b_{1}+b_{2}+b_{3}\right)+4 a_{1} \beta\left(b_{1}+b_{2}\right)\left(b_{1}+b_{2}+b_{3}\right) \\
= & \left(\beta\left(\left(a_{1}+a_{2}\right)\left(b_{1}+b_{2}+b_{3}\right)-\left(1-b_{1}\right)-\beta\left(b_{1}+b_{2}\right)\left(\sum_{i=1}^{3} a_{i}-N\right)\right)^{2}\right. \\
& +4\left(1-b_{1}\right) \beta\left(a_{1}+a_{2}\right)\left(b_{1}+b_{2}+b_{3}\right)+4 a_{1} \beta\left(b_{1}+b_{2}\right)\left(b_{1}+b_{2}+b_{3}\right)>0
\end{aligned}
$$

provided that $0 \leq \tau<1$ and $0 \leq \kappa<1$.

Thus both roots of the quadratic equation $A z^{2}+B z+C=0$ are real. Let the roots of the equation be $z_{1}$ and $z_{2}$. Then we have

$$
z_{1}=\frac{-B+\sqrt{B^{2}-4 A C}}{2 A} \text { and } z_{2}=\frac{-B-\sqrt{B^{2}-4 A C}}{2 A} .
$$

We will show shortly that under the condition $\mu<N /(2 N+n)$ we have $C<0$. . Let us assume that $C<0$ and therefore we have $A C<0$ as $A>0$. There are three cases to be discussed.

(i) If $B>0$, then $z_{1}>0, z_{2}<0$.

(ii) If $B<0$, then $z_{1}>0, z_{2}<0$.

(iii) If $B=0$, from $B^{2}-4 A C>0$, we know $A C<0$, then we have $z_{1}>0, z_{2}<0$.

In general, when $C<0$ we have

$$
z_{1}=\frac{-B+\sqrt{B^{2}-4 A C}}{2 A}>0
$$

being positive (the root has a practical meaning). Then we have the expression of $y, m, q, x$ in the equilibrium point:

$$
y=a_{1}+b_{1} z_{1} \quad m=a_{2}+b_{2} z_{1}, \quad q=a_{3}+b_{3} z_{1}, \quad x=N-y-m-q .
$$


Now the following is the condition we need to guarantee a unique positive root.

$$
C=\beta\left(a_{1}+a_{2}\right)\left(\sum_{i=1}^{3} a_{i}-N\right)-a_{1}<0 .
$$

In fact,

$$
\begin{aligned}
a_{2}=\frac{(1-\kappa) \tau n \mu}{\kappa+\frac{n}{N}-\frac{\kappa n}{N}}= & \frac{(1-\kappa) \tau n \mu N}{\kappa N+n-\kappa n}=\frac{(1-\kappa) \tau n \mu N}{\kappa(N-n)+n}=\frac{(1-\kappa) \tau \mu N}{\kappa \frac{(N-n)}{n}+1} \\
& \leq(1-\kappa) \tau \mu N \leq \tau \mu N
\end{aligned}
$$

and

$$
\begin{aligned}
\frac{\tau \mu \kappa(1-\kappa)\left(1-\frac{n}{N} N\right)}{\kappa+\frac{n}{N}-\frac{\kappa n}{N}}= & \frac{\tau \mu \kappa(1-\kappa)(N-n)}{\kappa+\frac{n}{N}(1-\kappa)}=\frac{\tau \mu(1-\kappa)(N-n)}{1+\frac{n}{N} \frac{1-\kappa}{\kappa}} \\
& <\tau \mu(1-\kappa)(N-n)<\tau \mu(1-\kappa) N .
\end{aligned}
$$

Thus, we get $a_{3} \leq \tau \kappa \mu N+\tau \mu(1-\kappa) N$. As a summary, we have

$$
\left\{\begin{array}{l}
a_{1}=(1-\tau) n \mu \\
a_{2}=\frac{(1-\kappa) \tau n \mu}{\kappa+\frac{n}{N}-\frac{\kappa n}{N} \leq \tau \mu N} \\
a_{3}=\tau \kappa \mu N+\frac{\tau \mu \kappa(1-\kappa)\left(1-\frac{n}{N}\right) N}{\kappa+\frac{n}{N}-\frac{\kappa n}{N}} \leq \tau \kappa \mu N+\tau \mu(1-\kappa) N .
\end{array}\right.
$$

Hence

$$
a_{1}+a_{2}+a_{3} \leq n \mu+2 \mu N
$$

Thus if we have

$$
\mu<\frac{N}{2 N+n}
$$

then

$$
\sum_{i=1}^{3} a_{i}-N<0
$$

and therefore $C<0$.

5.2. Proof of Proposition 3. It is obvious that the equilibrium point of (4) satisfies (5) or

$$
\left\{\begin{array}{l}
F=-y+a_{1}+b_{1} z=0 \\
G=-m+a_{2}+b_{2} z=0 \\
H=-q+a_{3}+b_{3} z=0 \\
K=-z+y+\beta x y+\beta x m=0
\end{array}\right.
$$


where the numbers $a_{1}, a_{2}, a_{3}, b_{1}, b_{2}, b_{3}$ are defined in (7). The Jacobian matrix of the above equations is given by

$$
J=\left[\begin{array}{cccc}
-1 & 0 & 0 & b_{1} \\
0 & -1 & 0 & b_{2} \\
0 & 0 & -1 & b_{3} \\
1+\beta x & \beta x & 0 & -1
\end{array}\right]
$$

The characteristic polynomial of $J$ is reduced to

$$
\begin{aligned}
\operatorname{Det}(\lambda I-J) & =(\lambda+1) \cdot \operatorname{Det}\left(\left[\begin{array}{ccc}
\lambda+1 & 0 & -b_{1} \\
0 & \lambda+1 & -b_{2} \\
-1-\beta x & -\beta x & \lambda+1
\end{array}\right]\right) \\
& =(\lambda+1)^{2}\left[(\lambda+1)^{2}-b_{1}-\left(b_{1}+b_{2}\right) \beta x\right] .
\end{aligned}
$$

Since $x=\frac{z-y}{\beta(y+m)}$ and $a_{1}, a_{2}, b_{1}, b_{2}>0$, it follows from (7) that

$$
\begin{aligned}
b_{1}+\left(b_{1}+b_{2}\right) \beta x & =b_{1}+\left(b_{1}+b_{2}\right) \beta \cdot \frac{z-y}{\beta(y+m)} \\
& =b_{1}+\left(b_{1}+b_{2}\right) \cdot \frac{z-b_{1} z-a_{1}}{\left(a_{1}+a_{2}\right)+\left(b_{1}+b_{2}\right) z} \\
& <b_{1}+\frac{z-b_{1} z-a_{1}}{z} \\
& <b_{1}+1-b_{1}=1 .
\end{aligned}
$$

From (25) and (24), we observe that all the eigenvalues of the Jacobian matrix $J$ are real and negative. By [14, Theorem 1.6], we know that the equilibrium point of (4) determined by (9) is asymptotically stable.

5.3. Proof of Proposition 5. From (15), it is easy to check that the equilibrium solution of (14) is determined by

$$
\left\{\begin{array}{l}
F_{i}^{o}=-y_{i}+a_{i 1}^{o}+b_{i 1}^{o} z_{i}=0 \\
G_{i}^{o}=-m_{i}+a_{i 2}^{o}+b_{i 2}^{o} z_{i}=0 \\
H_{i}^{o}=-q_{i}+a_{i 3}^{o}+b_{i 3}^{o} z_{i}=0 \\
K_{i}^{o}=-z_{i}+y_{i}+\beta_{i} x_{i} y_{i}+\beta_{i} x_{i} m_{i}=0
\end{array}\right.
$$

for $i=1,2, \ldots, s$. The Jacobian matrix of the above equations is given by

$$
J^{o}=\operatorname{diag}\left(J_{1}^{o}, \ldots, J_{s}^{o}\right), \quad J_{i}^{o}=\left[\begin{array}{cccc}
-1 & 0 & 0 & b_{i 1}^{o} \\
0 & -1 & 0 & b_{i 2}^{o} \\
0 & 0 & -1 & b_{i 3}^{o} \\
1+\beta_{i} x_{i} & \beta_{i} x_{i} & 0 & -1
\end{array}\right] .
$$

For $i=1, \ldots, s$, we can show that all the eigenvalues of $J_{i}^{o}$ are real and negative by following the similar proof of Proposition 3. Therefore, all the eigenvalues of the Jacobian matrix $J^{o}$ are real and negative. By [14, Theorem 1.6], we know that the equilibrium point of (14) is asymptotically stable. This completes the proof. 
5.4. Proof of Proposition 6. It is obvious that the equilibrium point for the network of $s$ prisons is determined by (16), i.e.,

$$
\left\{\begin{aligned}
F_{i}= & -y_{i}+a_{i 1}+b_{i 1} z_{i}+(1-\tau) \sum_{j=1, j \neq i}^{s} \frac{h}{N_{j}} z_{j}=0 \\
G_{i}= & -m_{i}+a_{i 2}+b_{i 2} z_{i}+e_{i} \sum_{j=1, j \neq i}^{s} \frac{h}{N_{j}} m_{j}+f_{i} \sum_{j=1, j \neq i}^{s} \frac{h}{N_{j}} z_{j}=0 \\
H_{i}= & -q_{i}+a_{i 3}+b_{i 3} z_{i}+g_{i} \sum_{j=1, j \neq i}^{s} \frac{h}{N_{j}} m_{j} \\
& +h_{i} \sum_{j=1, j \neq i}^{s} \frac{h}{N_{j}} q_{j}+w_{i} \sum_{j=1, j \neq i}^{s} \frac{h}{N_{j}} z_{j}=0 \\
K_{i}= & -z_{i}+y_{i}+\beta_{i} x_{i} y_{i}+\beta_{i} x_{i} m_{i}=0 .
\end{aligned}\right.
$$

The Jacobian matrix of the above equations has the following form

$$
J=\left[\begin{array}{cccc}
J_{11} & J_{12} & \cdots & J_{1 s} \\
J_{21} & J_{22} & \cdots & J_{2 s} \\
\vdots & \vdots & \ddots & \vdots \\
J_{s 1} & J_{s 2} & \cdots & J_{s s}
\end{array}\right]
$$

where

$$
J_{i i}=\left[\begin{array}{cccc}
-1 & 0 & 0 & b_{i 1} \\
0 & -1 & 0 & b_{i 2} \\
0 & 0 & -1 & b_{i 3} \\
1+\beta_{i} x_{i} & \beta_{i} x_{i} & 0 & -1
\end{array}\right]
$$

and

$$
J_{i j}=\left[\begin{array}{cccc}
0 & 0 & 0 & (1-\tau) \frac{h}{N_{j}} \\
0 & e_{i} \frac{h}{N_{j}} & 0 & f_{i} \frac{h}{N_{j}} \\
0 & g_{i} \frac{h}{N_{j}} & h_{i} \frac{h}{N_{j}} & w_{i} \frac{h}{N_{j}} \\
0 & 0 & 0 & 0
\end{array}\right] \quad \text { for } i \neq j
$$

From (16) and (15), it follows that for $1 \leq i \leq s$,

$$
\left\{\begin{array}{l}
b_{i 1}=b_{i 1}^{o}-(1-\tau)(s-1) \frac{h}{N_{i}} \\
b_{i 2}=b_{i 2}^{o}-\frac{(1-\kappa) \tau}{\left(\kappa+\frac{n_{i}}{N_{i}}-\frac{\kappa n_{i}}{N_{i}}\right) c_{i}}(s-1) \frac{h}{N_{i}} \equiv b_{i 2}^{o}-e_{i}^{s} \frac{h}{N_{i}} \\
b_{i 3}=b_{i 3}^{o}-\frac{\kappa \tau}{d_{i}\left(\kappa+\frac{\kappa i}{N_{i}}-\frac{\kappa n_{i}}{N_{i}}\right)}\left(\frac{N_{i}}{n_{i}}+\frac{\left(1-d_{i}\right)(1-\kappa)}{c_{i}}\right)(s-1) \frac{h}{N_{i}} \equiv b_{i 3}^{o}-f_{i}^{s} \frac{h}{N_{i}},
\end{array}\right.
$$

Then we have

$$
J_{i i}=J_{i}^{o}+W_{i}, \quad W_{i}=\left[\begin{array}{cccc}
0 & 0 & 0 & (1-\tau)(s-1) \frac{h}{N_{i}} \\
0 & 0 & 0 & e_{i}^{s} \frac{h}{N_{i}} \\
0 & 0 & 0 & f_{i}^{s} \frac{h}{N_{i}} \\
0 & 0 & 0 & 0
\end{array}\right], \quad 1 \leq i \leq s,
$$


where $J_{i}^{o}$ is defined in (26). Therefore, we can rewrite the Jacobian matrix $J$ as the form

$$
J=J^{o}+R
$$

where

$$
R=\left[\begin{array}{cccc}
W_{1} & J_{12} & \cdots & J_{1 s} \\
J_{21} & W_{2} & \cdots & J_{2 s} \\
\vdots & \vdots & \ddots & \vdots \\
J_{s 1} & J_{s 2} & \cdots & W_{s}
\end{array}\right]
$$

By Proposition 5, we know that all the eigenvalues of $J^{o}$ are real and negative if

$$
\mu<\frac{N_{i}}{2 N_{i}+n_{i}} \text { for } i=1,2, \ldots, s .
$$

By Theorem VIII.1.1 in [1], we have that for any two $n \times n$ matrices $E$ and $F$ with the eigenvalues $\phi_{1}, \ldots, \phi_{n}$ and $\psi_{1}, \ldots, \psi_{n}$, respectively,

$$
\max _{j} \min _{i}\left|\phi_{i}-\varphi_{j}\right| \leq(\|E\|+\|F\|)^{1-1 / n}\|E-F\|^{1 / n},
$$

where $\|\cdot\|$ denote any matrix norm. Now, suppose that the eigenvalues of the matrices $J$ and $J^{o}$ are denoted by $\lambda_{1}, \ldots, \lambda_{4 s}$ and $\lambda_{1}^{o}, \ldots, \lambda_{4 s}^{o}$, respectively. Then, for the matrix 1-norm $\|\cdot\|_{1}$, we get

$$
\begin{aligned}
\max _{j} \min _{i}\left|\lambda_{i}-\lambda_{j}^{o}\right| & \leq\left(\|J\|_{1}+\left\|J^{o}\right\|_{1}\right)^{1-1 /(4 s)}\|R\|_{1}^{1 /(4 s)} \\
& \leq\left(2\left\|J^{o}\right\|_{1}+\|R\|_{1}\right)^{1-1 /(4 s)}\|R\|_{1}^{1 /(4 s)},
\end{aligned}
$$

Next, we estimate $\|R\|_{1}$. Let

$$
\left\{\begin{aligned}
\xi_{j} & =\sum_{i=1, i \neq j}^{s}\left(e_{i}+g_{i}\right) \\
\zeta_{j} & =\sum_{i=1, i \neq j}^{s} h_{i} \\
\eta_{j} & =\sum_{i=1, i \neq j}^{s}\left(1-\tau+f_{i}+w_{i}\right)+(1-\tau)(s-1)+e_{j}^{s}+f_{j}^{s} \\
\delta & =\max _{j}\left\{\xi_{j}, \zeta_{j}, \eta_{j}\right\} .
\end{aligned}\right.
$$

Then

$$
\begin{aligned}
\|R\|_{1} & =\max _{j}\left\{\xi_{j} \frac{h}{N_{j}}, \zeta_{j} \frac{h}{N_{j}}, \eta_{j} \frac{h}{N_{j}}\right\} \\
& \leq \delta \max _{j} \frac{h}{N_{j}} \leq \delta
\end{aligned}
$$

Let

$$
\epsilon<\min \left\{1, \frac{\min _{j}\left|\lambda_{j}^{o}\right|^{4 s}}{\delta\left(2\left\|J^{o}\right\|_{1}+\delta\right)^{4 s-1}}\right\}
$$


By (28) and (27), we know that if $h$ is small such that $\max _{j} \frac{h}{N_{j}} \leq \epsilon$, then the real part of each eigenvalue $\lambda_{j}$ of the Jacobian matrix $J$ are negative. By [14, Theorem 1.6], it follows that the equilibrium point of the discrete model for the network of $s$ prisons is asymptotically stable.

\section{REFERENCES}

[1] R. Bнatia, Matrix Analysis, Springer-Verlag, New York, 1997.

[2] W. Ching, T. NG, And S. Chung, On Modeling SARS in Hong Kong, International Journal of Applied Mathematics, 13(2003), pp. 1-7.

[3] W. Ching, T. Ng, Y. Cong, And A. TAI, A Fast Algorithm for the Spread of HIV in a System of Prisons, Mathematical and Computer Modeling, 46(2007), pp. 1247-1255.

[4] D. Daley and J. Gani, Epidemic modelling: An Introduction, Cambridge University Press, Cambridge, 1999.

[5] S. Eubank, H. Guclu, V. Kumar, M. Marathe, A. Srinivasan, Z. Toroczkai, and N. WANG, Modelling Disease Outbreaks in Realistic Urban Social Networks, Nature, 429(2004), pp. 180-184.

[6] J. Gani, S. Yakowitz, And M. Blount, The Spread and Quarantine of HIV Infection in a Prisoner System, SIAM J. Appl. Math., 57(1997), pp. 1510-1530.

[7] G. Golub and C. van Loan, Matrix Computations, 2nd Edition, The John Hopkins University Press, London, 1989.

[8] D. Greenhalgh and F. Lewis, The General Mixing of Addicts and Needles in a Variableinfectivity Needle-sharing Environment, Journal of Mathematical Biology, 44(2002), pp. $561-598$.

[9] X. Huang and M. Villasana, An Extension of the Kermack-Mckendrick Model for AIDS Epidemic, Journal of the Franklin Institute, 342(2005), pp. 341-351.

[10] C. Kelly, Iterative Methods for Linear and Non-linear Equations, SIAM, Philadelphia, 1995.

[11] W. Ma, M. Song, and Y. Takeuchi, Global Stability of an SIR Epidemic Model with Time Delay, Appl. Math. Letters, 17(2004), pp. 1141-1145.

[12] M. Newman, The Structure and Function of Complex Networks, SIAM Review, 45(2003), pp. $167-256$.

[13] T. NG, G. Turinici, And A. Danchin, A Double Epidemic Model for the SARS Propagation, BMC Infectious Diseases, 3(2003), pp. 1-16.

[14] R. Seydel, Practical Bifurcation and Stability Analysis: From Equilibrium to Chaos, 2nd Edition, Springer-Verlag, New York, Berlin, Heidelberg, 1994.

[15] W. Wang, Global Behavior of an SEIRS Epidemic Model with Time Delays, Appl. Math. Letters, 15(2002), pp. 423-428. 